\title{
Stable Multi Optimized Algorithm Used For Controlling The Load Shedding Problems In Power Systems
}

\author{
Nila P Divakaran, A Dyaneswaran,M.E., \\ PG Scholar in Power Systems Engineering Vivekanandha College of Engineering for Women Elayampalayam, \\ Tamilnadu \\ Assistant Professor in EEE Dept, Vivekanandha College of Engineering for Women Elayampalayam, Tamilnadu
}

\begin{abstract}
This paper presents new approaches to update our existing load shedding scheme, planned for the coming years. If generation in power system is insufficient to power all loads, efficient load shedding operations may need to be deployed to maintain the supply demand balance. This paper proposes a distributed multi agent based PSO and GA, which can make the efficient load shedding decision based on the global information. This method integrates the multi agent system and PSO,GA algorithm. In order to obtain optimal solution quickly, each agent competes and corporate with its neighbours, and it can also learn by using its knowledge. According to this algorithm, total net active power and operating status of loads can be discovered accurately even with faults. For further improvement, can apply genetic algorithm for controlling purposes, compare both the methods.
\end{abstract}

Keywords: Multi Agent System, Particle swarm optimization, Genetic Algorithm, Load shedding, Voltage Stability

\section{INTRODUCTION}

The Objective of Power Systems designs are high power, electrical utility applications, industrial and commercial applications. Power Management is the aspect of managing the electrical loads such as available power loads are not overloaded The important objective of power systems design for high power, electrical utility applications, industrial and commercial applications. Power management is the aspect of (1) managing the electrical loads such as available power loads are not overloaded. (2) Power is allocated to the different loads such that the loads are receiving a proper allocation of power. Power management gives the good idea about Electrical Demand and Supply.

Power Management Systems (PMS) are essential for safe, efficient and reliable operation of industrial power systems. The functionality of the PMS suite includes load-shedding, power sharing, network synchronization and power restoration. PMS solutions protect and optimize the stability of industrial power systems in disturbance situations by ensuring power sharing between generators when the industrial power system is islanded from the grid. These solutions also ensure that the generators meet the required power demand when the network is connected to the grid. The load shedding functionality ensures power availability to critical process loads by dropping less critical loads.

The load shedding problem has a significant influence on secure and economic operation of power systems. Suppose any faults, sudden load change, and insufficient generation can create power mismatch between generation and loads. Load shedding is the process of tripping certain amount of load with lower priority to maintain the stability of the remaining portion of system.

Different methods have been proposed for load shedding. Multi Agent System is one of the most popular distributed control solutions. This control scheme requires the collection and transmission of global information. Advantages of MAS include the ability to survive single point failures and decentralized data processing, which leads to efficient task distribution. It causing faster operation and decision making process

The Multi Agent system and Particle Swarm Optimization are integrated to form the proposed MAPSO method for solving load shedding problem. In MAPSO not only a solution to the optimization problem but also a particle to PSO. According to this method, two agents will communicate with each other only if their corresponding buses are connected. Through information exchanges, each agent can discover necessary global information for load shedding decision making. PSO is used to optimize the information exchange. Then the load shedding activities of all agents can be coordinated. Find out the optimum value of the load to be shed.

Genetic Algorithm (GA) is a global optimization algorithm derived from evolution and natural selection. Although genetic algorithm cannot always provide optimal solution, it has its own advantages (Liu yong, Kang lishan \& Chen yuping. 1997) and is a powerful tool for solving complex problems (Xi yugeng, Chai tianyou \& Yun weimin. 1996). 
The basic thought of Genetic algorithm:

1) Randomly producing a original population whose number of individuals is a constant $\mathrm{N}$.

2) Producing next generation by crossing over and mutation among individuals.

3) Forming the new population of $\mathrm{N}$ individuals from the generation

4) Producing the next population by repeating the step2

Multi Agent Based PSO is used for controlling the load shedding problem. Faults, sudden load change and insufficient generation can create major problems between generation and loads. If generation is insufficient, efficient load shedding operations may need to be maintaining the supply demand balance. It focuses interactions between a utility company and its customers/users. This is the Diagram for the ILS sceme



Figure 1 complete load shedding scheme

This method proposed to determine the most appropriate loads to be shed during under frequency and under voltage condition. Total net active power can be calculating and operating status of loads can be estimating accurately even with faults. It based on the discovered information; coordinated Load shedding decision can be made

\section{Problem Formulation}

Load shedding problem can be formulated as an optimization problem with the following objective function and constraints:

Objective function:

The objective function (OBF) of the load shedding problem is to minimize the sum of curtailed load during generation outage conditions. It can be expressed mathematically as:

$$
\min \mathrm{f}(\mathrm{t})=\sum_{\mathrm{i}=1}^{\text {Nbus }}\left(\alpha \text { i. } \Delta \mathrm{pdi}^{2}+\beta \text { i. } \Delta \mathrm{qdi}^{2}\right)
$$

Where $\alpha i$ and $\beta i$ are the weight factors for curtailed active and reactive power load of the $i$ th bus and Nbus is the number of buses in the transmission system. $\Delta$ Pdi and $\Delta$ Qdi are the curtailed active and reactive power load of the $i$ th transmission system.

Constraints:

The constraints can be listed as follows:

- Power flow balance equations:

$$
\begin{array}{r}
\text { Pgi }- \text { Pdio }-\Delta \text { pdi- Vi } \sum_{\mathrm{J}=1}^{\mathrm{N}} \mathrm{Vj} \text { Yij } \cos (\delta \mathrm{i}-\delta \mathrm{j}-\theta \mathrm{ij})=0 \ldots \ldots(2) \\
\mathrm{N} \\
\text { Qgi }- \text { Qdio }-\Delta \text { Qdi- Vi } \sum_{\mathrm{J}=1} \mathrm{Vj} \text { Yij } \cos (\delta \mathrm{i}-\delta \mathrm{j}-\theta \mathrm{ij})=0 \ldots \ldots(3)
\end{array}
$$

$P g i$ and $Q g i$ are active and reactive power generations at the $i$ th bus. $P d i 0$ and $Q d i 0$ are initial active and reactive power load of the $i$ th bus. $V$ 's and $\delta$ 's, are system bus voltages magnitudes and phase angles. $Y i j$ and $\theta i j$ are bus admittance matrix elements.

- Maintaining the original load power factor:

To maintain the original load power factor $\Delta Q d i$ is selected as: 
$\Delta \mathrm{Qdi}=\Delta \mathrm{Pdi}[\Delta \mathrm{Q}$ dio $/ \Delta \mathrm{Pdio}]$

- Generators active and reactive power limits:

$\mathrm{Pgi}^{\min } \leq \mathrm{Pgi} \leq \mathrm{Pgi}^{\mathrm{max}} \quad \mathrm{i}=1 \ldots$ No.

$\mathrm{Qgi}^{\mathrm{min}} \leq \mathrm{Qgi}^{\mathrm{L}}<\mathrm{Qgi}^{\mathrm{max}}$

$\mathrm{i}=1 \ldots$ No

- Voltage range limits:

$\mathrm{Vi}^{\min } \leq \mathrm{Vi} \leq \mathrm{Vi}{ }^{\max }$

- Line loading limits:

$|\delta \mathrm{i}-\delta \mathrm{j}| \leq \varepsilon \mathrm{ij}$

Where $\delta i$ and $\delta \mathrm{j}$ are the voltage angles at bus $i$ and bus $j$, and $\varepsilon i j$ is the maximum voltage phase angle difference between buses $i$ and $j$.

\section{Multi Agent Based Particle SWARm Optimization And Genetic Algorithm}

\section{A. Particle Swarm Optimization}

PSO simulates the behaviors of bird flocking. Suppose the following scenario: a group of birds are randomly searching food in an area. There is only one piece of food in the area being searched. All the birds do not know where the food is. But they know how far the food is in each iteration. The effective one is to follow the bird, which is nearest to the food. PSO learned from the scenario and used it to solve the optimization problems.

PSO is initialized with a group of random particles and then searches for optima by updating generations. In every iteration, each particle is updated by following two "best" values. The first one is the best solution (fitness) it has achieved so far. (The fitness value is also stored.) This value is called pbest. Another "best" value that is tracked by the particle swarm optimizer is the best value, obtained so far by any particle in the population.

This best value is a global best and called g-best. When a particle takes part of the population as its topological neighbors, the best value is a local best and is called p-best. After finding the two best values, the particle updates its velocity and positions with following equation.

$\mathrm{Vi}(\mathrm{u}+1)=\mathrm{w} * \mathrm{Vi}(\mathrm{u})+\mathrm{C} 1 *$ rand ()$*($ pbest $\mathrm{i}-\mathrm{Pi}(\mathrm{u}))+\mathrm{C} 2 *$

rand ()$^{*}($ gbesti $-\mathrm{Pi}(\mathrm{u}))$

$\mathrm{Pi}(\mathrm{u}+1)=\mathrm{Pi}(\mathrm{u})+\mathrm{Vi}(\mathrm{u}+1)$

In the above equation,

The term rand ( )*(pbesti $-\mathrm{Pi}(\mathrm{u}))$ is called particle memory influence

The term rand ()$*($ gbesti $-\mathrm{Pi}(\mathrm{u}))$ is called swarm influence

$\mathrm{Vi}(\mathrm{u})$ which is the velocity of ith particle at iteration ' $u$ ' must lie in the range

$$
\operatorname{Vmin} \leq \operatorname{Vi}(\mathrm{u}) \leq \mathrm{Vmax}
$$

- The parameter Vmax determines the resolution, or fitness, with which regions are to be searched between the present position and the target position

- If Vmax is too high; particles may fly past good solutions. If Vmin is too small, particles may not explore sufficiently beyond local solutions.

- In many experiences with PSO, Vmax was often set at 10-20\% of the dynamic range on each dimension.

- The constants C1and C2 pull each particle towards pbest and gbest positions.

- Low values allow particles to roam far from the target regions before being tugged back. On the other hand, high values result in abrupt movement towards, or past, target regions. 
- The acceleration constants $\mathrm{C} 1$ and $\mathrm{C} 2$ are often set to be 2.0 according to past experiences

- Suitable selection of inertia weight ' $\omega$ ' provides a balance between global and local explorations, thus requiring less iteration on average to find a sufficiently optimal solution.

- In general, the inertia weight $w$ is set according to the following equation,

$\mathrm{W}=\mathrm{W}_{\max }-\left[\mathrm{W}_{\max }-\mathrm{W}_{\min } / \mathrm{ITER}_{\max }\right] * \mathrm{ITER}$

Where $\mathrm{w}$-is the inertia weighting factor

Wmax - maximum value of weighting factor

Wmin - minimum value of weighting factor

ITERmax - maximum number of iterations

ITER - current number of iteration

The PSO algorithm can be best described in general as follows:

1) For each particle, the position and velocity vectors will be randomly initialized with the same size as the problem dimension.

2) Measure the fitness of each particle (pbest) and store the particle with the best fitness (gbest) value.

3) Update velocity and position vectors for each particle.

4) Repeat steps until a termination criterion is satisfied.

On the other hand, some advantages of aforementioned algorithms over PSO are the following:

- The availability of commercial versions of some algorithms like Matlab (genetic algorithm) and Excel premium solver (evolutionary programming).

- The extensive collection of books and research literatures, especially in the case of genetic algorithm and evolutionary programming, which cover these competing methods. Despite the simplicity of the PSO concept and implementation, its superiority is proven when compared with other techniques in many different application areas.

\section{B. Genetic Algorithm}

Genetic algorithm is a search method that employs processes found in natural biological evolution. These algorithms search or operate on a given population of potential solutions to find those that approach some specification or criteria. To do this, the genetic algorithm applies the principle of survival of the fittest to find better and better approximations. At each generation, a new set of approximations is created by the process of selecting individual potential solutions (individuals) according to their level of fitness in the problem domain and breeding them together using operators borrowed from natural genetics. This process leads to the evolution of population of individuals that are better suited to their environment than the individuals that they were created from, just as in natural adaptation.

\section{Multi agent System}

Agents have a certain level of autonomy, which means that they can take decisions without a central controller or commander. To achieve this, they are driven by a set of tendencies. For a battery system a tendency could be: "charge the batteries when the price for the $\mathrm{kWh}$ is low and the state of charge is low, too". Thus, the MAS decide when to start charging based on its own rules and goals and not by an external command. In addition, the autonomy of every agent is related to the resources that it possesses and uses. These resources could be the available fuel for a diesel generator.

Another significant characteristic of the agents is that they have partial or none at all representation of the environment. For example, in a power system the agent of a generator knows only the voltage level of its own bus and, maybe, it can estimate what is happening in certain specific buses. However, the agent does not know what is happening in the whole system. This is the core of the MAS technology, since the goal is to control a very complicated system with minimum data exchange and minimum computational demands.

Finally, another significant characteristic is that an agent has a certain behavior and tends to satisfy certain objectives using its resources, skills and services. An example of these skills could be the ability to produce or store power and an example for the services could be the ability to sell power in a market. The way that the agent uses the resources, skills and services characterizes its behavior. As a consequence, it is obvious that the behavior of every agent is formed by its goals. An agent that controls a battery system and its goal is to supply uninterruptible power to a load will have different behavior from a similar battery, whose primary goal is to maximize profits by bidding in the energy market.

MAS technology provides an opportunity to compute and optimize many complicated problems. Agents in MAS act collectively as a society and they collaborate (or compete) to achieve their own individual 
goals as well as the common goal. This cooperative and competitive feature matches the essential nature of a particle in PSO. Hence, this paper combines PSO and MAS to form a new optimal algorithm.

In general, the following four elements should be defined when MAS is used to solve problems:

a) The meaning and the purpose of each agent in MAS;

b) An environment where all agents live;

c) The definition of a local environment;

d) A set of behavioral rules, governing the interaction between the agents and their environment. They are the laws of the agent universe.

\section{Multi Agent based PSO and Genetic Algorithm}

Enlightened by multi agent system and PSO, this paper integrates multi agent system and PSO to form a multi agent-based PSO approach (MAPSO), for solving the optimization problem. In MAPSO, an agent represents a particle to PSO and a candidate solution to the optimization problem. All agents live in a lattice-like environment, with each agent fixed on a lattice point.

In order to obtain optimal solution quickly, they compete and cooperate with their neighbors, and they can also use knowledge. Making use of these agent-agent interactions and evolution mechanism of PSO in a lattice-like environment, the proposed method can find high-quality solutions reliably with the faster convergence characteristics in a reasonably good computation time.

In this study, MAS and PSO are integrated to form the proposed MAPSO method for solving reactive power optimization dispatch. In MAPSO, an agent represents not only a candidate solution to the optimization problem but also a particle to PSO. Firstly, a lattice-like environment is constructed, with each agent fixed on a lattice-point.

Multi Agent based Genetic Algorithms (MAGA) have recently received much attention as robust stochastic search algorithms for optimization problems. GAs are blind search technique using stochastic operations based on the mechanics of the survival of the fittest. It also works with a population of individuals rather than single point. Operation, involve random number generation (mutation), string copying (reproduction), and partial string exchange (crossover). Each string represents a possible solution. It starts with the formulation of the "fitness function", which represents the objective function for the problem. Based on the fitness of the population strings, two parent strings are selected probabilistically in the reproduction process. Two child strings are then generated from the parent strings in the process of crossover by complementing the child strings at selected bit positions.

In order to obtain optimal solution quickly, each agent competes and cooperates with their neighbors, and they can also use knowledge to obtain high-quality optimal solution by self-learning. Making use of evolution mechanism of PSO, it can speed up the transfer of information among agents, and the proposed MAPSO method can realize the purpose of optimizing the value of objective function.

: In MAPSO, an agent represents a candidate solution to the optimization problem in hand and is a particle to PSO. Hence, agent has a fitness value to the optimization problem. For solving reactive power problem, its fitness value is the value of the active power loss in the transmission network

$$
\mathrm{f}(\alpha)=\mathrm{f}_{\mathrm{Q}}=\sum_{\mathrm{k}} \underset{\varepsilon \mathrm{N}_{\mathrm{R}}}{\mathrm{P}_{\text {kloss }}}=\underset{\mathrm{k} \varepsilon \mathrm{N}_{\mathrm{R}}}{\mathrm{gk}}\left(\mathrm{Vi}^{2}+\mathrm{Vj}^{2}-2 \mathrm{Vi} \mathrm{Vj} \cos \theta \mathrm{ij}\right)
$$

The purpose of is to minimize the real power transmission losses and keep all the voltages within the limits as much as possible. In reactive power optimization problem, each agent carries all control variables to be optimized.

In MAS, all agents live in an environment. An environment is organized as a lattice-like structure in Fig. In the environment, each agent is fixed on a lattice-point and each circle represents an agent; the data in circle represents its position in the environment. Due to representation of a particle in PSO, each agent in its database contains two data, i.e., particle's current velocity and its coordinates in the search space.

Since each agent can only sense its local environment in MAS, the definition of the local environment is very important in the proposed method.

The neighbors of $\alpha \mathrm{ij}$ and $\mathrm{Nij}$ is defined as

Where

$$
\mathrm{N}_{\mathrm{i}, \mathrm{j}}=\left\{\alpha_{\mathrm{i}} 1, \mathrm{j}, \alpha_{\mathrm{i}, \mathrm{j}} 1, \alpha_{\mathrm{i}} 2, \mathrm{j}, \alpha_{\mathrm{i}, \mathrm{j}} 2\right\}
$$

$$
\begin{aligned}
& i^{1}=i-1 \quad i \neq 1, \\
& \mathrm{~L}_{\text {size }} \mathrm{i}=1 \\
& j^{1}=\begin{array}{l}
j-1 \quad j \neq 1 \\
L_{\text {size }} j=1
\end{array}
\end{aligned}
$$




$$
\begin{aligned}
\mathrm{i}^{2}=\mathrm{i}+1 \quad \mathrm{i} \neq \mathrm{L}_{\text {size }}, & \mathrm{j}^{2}=\begin{array}{c}
\mathrm{j}+1 \quad \mathrm{i} \neq \mathrm{L}_{\text {size }} \\
1 \quad \mathrm{i}=\mathrm{L}_{\text {size }}
\end{array} \quad \mathrm{i}=\mathrm{L}_{\text {size }}
\end{aligned}
$$

To quickly and accurately achieve its purposes, each agent has some behaviors. In MAPSO, each agent firstly competes and cooperates with its neighbors to diffuse its useful information to the whole environment, and it can also use evolution mechanism of PSO and its knowledge. On the basis of such behaviors, three operators are designed for the agents

\section{MAPSo AND Ga BASEd LoAd ShedDing STRATEgy}

As mentioned in previous sections, some load could be shed in emergency condition to protect the other load. But it is difficult for dispatcher to determine which load and how many could be shedding. Because load shedding bring up large economic lost, dispatcher must try his best to protect majority load. Therefore, an algorithm of load shedding could be a great help to the dispatcher. It is noticed that the penalty function in PSO could be considered as the load shedding in a power system which is the penalty for maintaining the safe operation of the power system, and the load shedding strategy can be optimized with the PSO.

The mathematical model of this new strategy is:

$$
\begin{aligned}
& \min f(P, Q)=\sum_{i \varepsilon 1} P_{g i}-\sum_{j \in L} P_{1 j}+\sum \mathrm{W}_{\mathrm{lj}}\left(\mathrm{P}_{\mathrm{lj}}-\mathrm{P}_{\mathrm{lj}}{ }^{\prime}\right) \ldots \ldots \ldots(16) \\
& \text {. } \quad \mathrm{P}_{\mathrm{i}}=\mathrm{Vi} \sum_{\mathrm{i}=1}^{\mathrm{n}} \mathrm{Vj}(\mathrm{Gij} \cos \theta \mathrm{ij}+\mathrm{Bij} \sin \theta \mathrm{i}) \text {. } \\
& \mathrm{n} \\
& \text {. } \quad \mathrm{Q}_{\mathrm{i}}=\mathrm{Vi} \sum_{\mathrm{i}=1} \mathrm{Vj}(\mathrm{Gij} \sin \theta \mathrm{ij}-\mathrm{Bij} \cos \theta \mathrm{i}) \\
& \mathrm{Pgi}^{\mathrm{min}} \leq \mathrm{Pgi} \leq \mathrm{Pgi}{ }^{\max } \\
& \mathrm{Qgi}^{\mathrm{min}} \leq \mathrm{Qgi} \leq \mathrm{Qgi}{ }^{\mathrm{max}} \\
& \mathrm{V} \min \leq \mathrm{Vi} \leq \mathrm{V} \max \\
& -\mathrm{Fi}^{\min } \leq \mathrm{Fi} \leq \mathrm{Fii}^{\max } \\
& 0 \leq \mathrm{P}_{\mathrm{lj}}{ }^{\prime} \leq \mathrm{P}_{\mathrm{lj}}
\end{aligned}
$$

It must be pointed that the reactive load always variable with the active load. In this paper, the reactive load will decrease with active load with the power factor remaining unchanged. While the equality constraints could be eliminated as explained in previous section, some of the inequality constraints could be handled easier than being added to the aim function as a penalty function. It is also noted that generator outputs and the loads are control variables, and they could be bounded after updating the position of particles.

The control variable P and Q not only contains the output of generator but also the active load. Plj is demand power at bus $\mathrm{j}$, but only $\mathrm{Plj}$ ' is supplied, so the amount of load Plj-Plj' is shed, the factor wlj is the economical lost coefficient for the load $\mathrm{j}$. It must be pointed that the reactive load always variable with the active load. In this paper, the reactive load will decrease with active load with the Power factor remaining unchanged. Suppose $x$ is the control variable vector consisting of $P$ and $Q$, etc., and it's dimension is $x n$. For particle $i$,

Let

$$
\begin{aligned}
& x_{i=}\left(x_{i}{ }^{1}, x_{i}{ }^{2}, \ldots . x_{i}{ }^{n}\right) \\
& x_{i, t+1}=x_{i, t}+v_{i, t+1} \ldots
\end{aligned}
$$

Then it becomes 


$$
\begin{aligned}
x_{\text {min }}^{\mathrm{j}} & \text { if } \mathrm{x}_{\mathrm{i}}^{\mathrm{j}}<\mathrm{x}_{\text {min }}^{\mathrm{j}} \\
\mathrm{x}_{\mathrm{i}}{ }^{1}=\mathrm{x}_{\text {max }}^{\mathrm{j}} & \text { if } \mathrm{x}_{\mathrm{i}}^{\mathrm{j}}>\mathrm{x}_{\text {max }}^{\mathrm{j}} \\
\mathrm{x}_{\mathrm{i}}^{\mathrm{j}} & \text { others }
\end{aligned}
$$

The power equation becomes

$$
\begin{aligned}
\min F(x)= & \sum_{i \varepsilon G} P g i-\sum_{j} \& L \\
& +\mathrm{w}_{\mathrm{h}} \sum_{\mathrm{J}=1} \max \left(\left(0, \mathrm{~h}_{\mathrm{j}}(\mathrm{x})\right)\right. \\
& +\sum \mathrm{w}_{\mathrm{lj}}\left(\mathrm{Plj}-\mathrm{plj}{ }^{\prime}\right)
\end{aligned}
$$

It can be seen that the aim function consists of three parts. The first part is the power loss; the second part is the penalty of violation of constraints; and the third part is the economic cost of load shedding. The inequality constraint now only contains voltage constraint and transmission capacity constraint.

The setting of penalty factor as above is to make a masking effect. The particle firstly flies to position where no constraint is violated, because the penalty to constraint violation is highest. Secondly, the particle searches the position where no load is shedding. And at last, the particle tries to decrease the power loss.



Figure 2 Flow chart

\section{IMPLEMENTATION OF MAPSO AND GENETIC ALgORITHM For LOAD SHEDDING PROBLEM}

In MAPSO and GA, many different operators are utilized to simulate the behaviors of agents, and realize their purposes. In order to reduce the computational cost, the self-learning operator is only performed on the agent with the minimum fitness value in each generation, but it has an important effect on the performance 
of MAPSO and GA. The proposed method can quickly and accurately converge to the optimal solutions for reactive power optimal dispatch. The details of the overall algorithm are as follows.

Step 1) Input parameters of system and algorithm, and specify the lower and upper boundaries of each variable.

Step 2) Generate a lattice-like environment, and initialize randomly each agent.

Step 3) Evaluate the fitness value of each agent based on the Load Shedding analysis results and the proposed mixed-variable handing methods.

Step 4) Update the time counter.

Step 5) Perform the neighborhood competition and cooperation operator on each agent.

Step 6) Execute the PSO operator and further adjust its position in the search space on each agent according to the velocity equations.

Step 7) Evaluate the fitness value of each agent based on the Load Shedding analysis results and the proposed mixed-variable handing methods.

Step 8) Find the best agent with the minimum fitness value, and then perform the self-learning operator.

\section{Simulation STUdies}

In this paper Multi Agent Based PSO and GA are used for controlling the Load shedding problems. The Load Shedding is assigned with the priority of customers and make the generation is 1000MW. Consider each and every agent they have different priorities First assign the lower priority, obtained the corresponding optimized Load value for the proper Load Shedding.

The consumers are represented as $\mathrm{C} 1, \mathrm{C} 2, \mathrm{C} 3, \mathrm{C} 4$, and $\mathrm{C}$, each consumer have different priority. It may be lower, medium or high, consider these priorities and got the optimum value corresponding to their Load Demand.

Total cost of Each Generation unit

$$
\begin{aligned}
& \text { total_cost }=2.6333 \mathrm{e}+003 \\
& \text { total_cost }=4.3752 \mathrm{e}+003 \\
& \text { total_cost }=1.3734 \mathrm{e}+004 \\
& \text { total_cost }=2.2673 \mathrm{e}+004 \\
& \text { total_cost }=3.7808 \mathrm{e}+004
\end{aligned}
$$

The Load Schedule corresponding to the generation

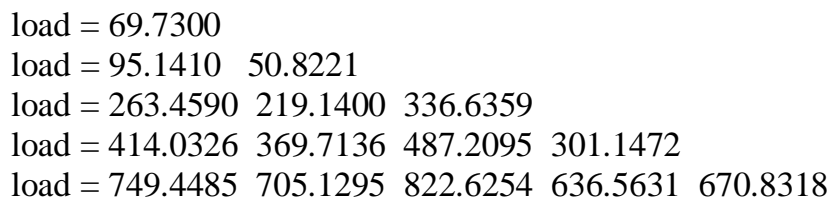

The Load Shedding is assigned with the priority of customers and make the generation is 1000MW. Consider each and every agent they have different priorities First assign the lower priority, obtained the corresponding optimized Load value for the Load shedding.

Obtained the total cost of each generation unit of the entire system and Corresponding Load Schedule is obtained. There are two cases, one is the system with losses and another one is system without losses. In both cases should get the total cost and Load Schedule. In this paper result represents the values without losses, so the error value is zero.

The consumers are represented as $\mathrm{C} 1, \mathrm{C} 2, \mathrm{C} 3, \mathrm{C} 4$, and $\mathrm{C} 5$, each consumer have different priority. It may be lower, medium or high, consider their priorities and got the optimum value of their corresponding Load Schedule. 


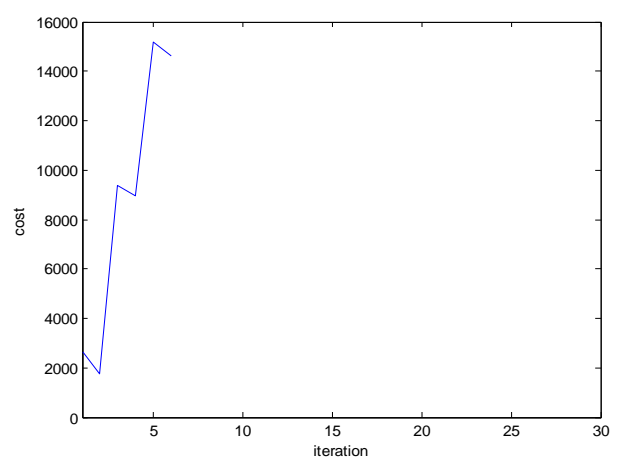

Figure 3 Cost Vs Iteration (30) without loss

This graph is plotted between scheduling cost and its irrespective iteration. In this method 3 generating units are committed along with the system. This plot shows the optimum value of the fuel cost value. Number of iteration is 30 . If the number of iteration is 60 , the graph shown below



Figure 4 Load Vs Iteration (without loss)

This graph shows the graph between load and respective iteration. The Load Shedding is assigned with the priority. Make the generation is $1000 \mathrm{MW}$. There are different priorities assigned with each agent. First consider the lower priority, got the corresponding optimized Load value for the Load shedding. The graph between net power and iteration shown below. Sometimes the load value is taken as corresponding net power of each agent.

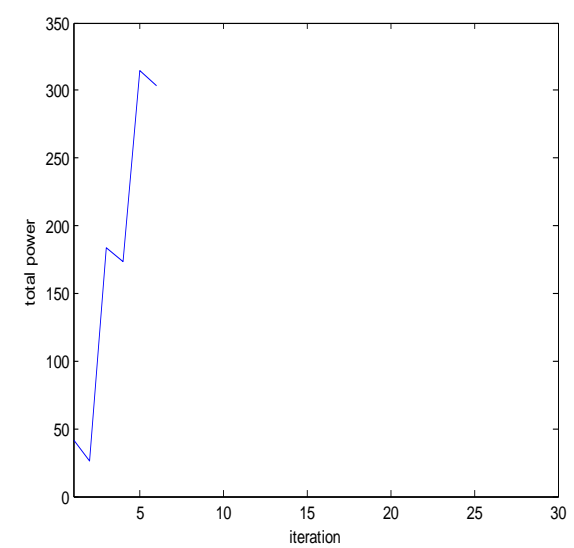

Figure 5 Total net Power Vs Iteration 


\section{REFERENCES}

[1] F. Shokooh, J. J. Dai, S. Shokooh, J. Taster, H. Castro, T. Khandelwal, and G. Donner, “An intelligent load shedding (ILS) system application in a large industrial facility," in Proc. 14th IAS Annu. Meeting, 2005, vol. 1, pp. 417-425.

[2] S. Borg, G. Andersson, D. J. Hill, and I. A. Hiskens, "On influence of load modeling for under voltage load shedding studies," IEEE Trans. Power Syst., vol. 13, no. 2, pp. 395-400, May 1998.

[3] B. Otomega,M. Glavic, and T. Van Cutsem, "Distributed under voltage load shedding," IEEE Trans. Power Syst., vol. 22, no. 4, pp. 2283-2284, Nov. 2007.

[4] V. V. Terzija, "Adaptive under frequency load shedding based on the magnitude of the disturbance estimation," IEEE Trans. Power Syst., vol. 21, no. 3, pp. 1260-1266, Aug. 2006.

[5] B. Hamid, O. Abderrahmane, G. Nadir, M. Farid, and E. M. Nikos, "A new approach applied to adaptive centralized load shedding scheme," in Proc. 8th WSEAS Int. Conf., 2009, pp. 28-33.

[6] H. Seyedi and M. Sanaye-Pasand, "New centralized adaptive load-shedding algorithms to mitigate power system blackouts," IET Gen., Transm., Distrib., vol. 3, no. 1, pp. 99-114, 2009.

[7] M. Wooldridge, An Introduction to Multiagent Systems. New York: Wiley, 2002.

[8] J. A. Momoh and O. S. Diouf, "Optimal reconfiguration of the navy ship power system using agents," in Proc. IEEE PES,Transmission and Distribution Conf. Exhib., 2006, pp. 562-567.

[9] K. L. Butler, N. D. Sarma, and P. V. Ragendra, "Network reconfiguration for service restoration in shipboard power distribution systems," IEEE Trans. Power Syst., vol. 16, no. 4, pp. 653-661, Nov. 2001.

[10] T. L. Baldwin and S. A. Lewis, "Distribution load flow methods for shipboard power systems," IEEE Trans. Ind. Appl., vol. 40, no. 5, pp. 1183-1190, Sep./Oct. 2004.

[11] L. Sun and D. A. Cartes, "Reconfiguration of shipboard radial power system using intelligent agents," in Proc. ASNE Elect. Tech. Symp., Jan. 2004.

[12] J. M. Solanki, S. Khushalani, and N. N. Schulz, "A multi-agent solution to distribution systems restoration," IEEE Trans. Power Syst., vol. 22, no. 3, pp. 1026-1034, Aug. 2007.

[13] K. Huang, D. A. Cartes, and S. K. Srivastava, "A multiagent-based algorithm for ring-structured shipboard power system reconfiguration," IEEE Trans. Syst., Man, Cybern. C, Appl. Rev., vol. 37, no. 5, pp. 1016-1021, Sep. 2007.

[14] F. Xiao, L. Wang, and Y. Jia, "Fast information sharing in networks of autonomous agent," in Proc. American Control Conf., 2008, pp. 4388-4393. 